\title{
A dynamic two-zone model of continuous fluidized bed layering granulation with internal product classification
}

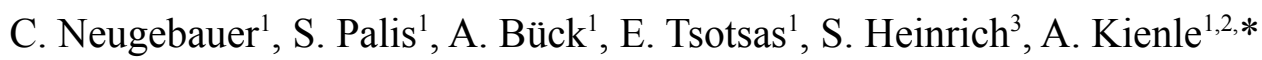 \\ 'Otto von Guericke University, Universitätsplatz 2, D-39106 Magdeburg, Germany \\ ${ }^{2}$ Max Planck Institute for Dynamics of Complex Technical Systems, Sandtorstraße 1, D-39106 \\ Magdeburg, Germany \\ ${ }^{3}$ Hamburg University of Technology, Denickestraße 15, D-21073 Hamburg, Germany \\ *Corresponding author. Fax: +49-391-6110-515; E-mail: kienle@mpi-magdeburg.mpg.de
}

\begin{abstract}
A dynamic two-zone model is proposed to address the formation of granulation and drying zones in fluidized bed layering granulation processes with internal product classification. The model assumes a constant volume for the granulation zone, but a variable overall volume for the fluidized bed to account for classified product removal. The model is used to study the effect of various process parameters on dynamics and process stability. Stability is shown to depend on the separation diameter of product removal and the flow rate of the injected liquid. A lower and upper range of separation diameters with stable process behavior are found. In an intermediate range instability in the form of selfsustained oscillations is observed. The lower stability boundary is in qualitative agreement with recent experimental observations (Schmidt et al., 2015a); the upper boundary was reported in a theoretical paper by Vreman et al. (2009) based on a single zone model.
\end{abstract}

Keywords: Layering granulation; Zone formation; Internal product classification; Population balance modeling; Stability analysis

\section{Introduction}

Fluidized bed layering granulation (FBLG) processes play an important role in chemical, pharmaceutical, and food industries. They are used to generate dust-free and free-flowing granules, which are usually more durable and easier to handle than their liquid equivalents (Heinrich, Peglow, Ihlow, Henneberg, \& Mörl, 2002a; Mörl, Heinrich, \& Peglow, 2007). Despite their importance, the dynamics of these processes remain poorly understood; a fundamental understanding is a prerequisite for stable process operation and the production of particles with tailor made properties.

In FBLG processes a suspension or solution is sprayed into a process chamber. The process chamber contains a large number of particles which are fluidized by a heated gas flow. The surface of the particles is wetted by the injected material, which leads, after drying, to an 'onion layer-wise' particle growth.

Industrial FBLG processes with high production rates are operated continuously. This requires a continuous supply of new nuclei to the granulation chamber. Besides an external supply of nuclei, two different mechanisms can lead to the generation of new nuclei. In processes with external product classification, this can be achieved by a continuous separation of oversized particles, which are ground with a mill and then recycled to the granulation chamber with the 
undersized particles (Heinrich et al., 2002a). These kinds of processes are usually operated with constant bed mass inside the granulation chamber.

In processes with internal product classification, an outlet tube is installed in the lower area of the process chamber (see Fig. 1). By means of an adjustable counter-current classification gas flow, the size of particles, which are withdrawn from the granulation chamber, can be adjusted. Internal formation of new nuclei can be achieved by suitable injection of liquid suspension leading to overspray, which after drying gives rise to new nuclei. As the outlet parameters are fixed to achieve a product size spectrum, bed mass and volume usually vary during operation (Vreman et al., 2009).

Fig.1

Both process configurations tend to be unstable for certain operating conditions. While the particle size distribution of the outlet remains almost constant, these instabilities can lead to selfsustained oscillations of the outlet mass flow, and therefore to variations of the hold-up in the apparatus. Although this problem was already known to practitioners for some time (Schütte, Ruhs, Pelgrims, Klasen, \& Kaiser, 1998), a rigorous experimental validation was given only recently by Schmidt, Rieck, Bück, and Tsotsas (2015b) for processes with external product classification, and by Schmidt, Bück, and Tsotsas (2015a) for processes with internal product classification.

A preliminary model based analysis of the instability of FBLG processes with external product classification was given by Heinrich, Peglow, and Mörl (2002b) and Radichkov et al. (2006), and for processes with internal product classification by Vreman, Van Lare, and Hounslow (2009). In these models, uniform particle growth was assumed. However, this is in contradiction to more recent experimental findings for batch processes where a widening of the particle size distribution over time has been observed. This phenomenon can be explained by zone formation (Hoffmann, Peglow, \& Tsotsas, 2011; Silva, Tamiozzo, Duarte, Murata, \& Barrozo, 2011; Peglow et al., 2014).

Fig. 1 illustrates that only part of the particles in the granulation chamber are wetted by the injected solution, giving rise to distinct granulation and drying zones. The influence of zone formation on the dynamics and stability of FBLG processes with external product classification was recently investigated by Dreyschultze et al. (2015), and further analyzed by Bück et al. (2016). The present study reveals the effect of zone formation on the dynamics of FBLG processes with internal product classification. A mathematical model is developed. In contrast to the external product classification process, variable bed mass has to be accounted for and combined with the effect of zone formation. The model is used for numerical bifurcation and stability analysis, and the results are compared with previous theoretical results (Vreman et al., 2009; Palis \& Kienle, 2013) and experimental findings (Schmidt et al., 2015a).

\section{Mathematical model}


The nomenclature and model structure of the FBLG process with internal product classification, as illustrated in Fig. 1, are introduced in Fig. 2.

Fig. 2

According to Figs. 1 and 2 the process chamber is divided into two functional zones. In the first zone, the granulation zone indicated by index 1, the spherical, non-porous particles, , are sprinkled with a liquid suspension or solution (with a volume flow rate ) and growth of the particles takes place. The second zone, indicated by index 2, is the drying zone. Within this zone, the particles are not in contact with the spray, and drying particles, , appear. Exchange rates between the granulation and the drying zones in Fig. 2 follow from the amount of particles in the respective zones and characteristic time constants and .

Following the arguments in Vreman et al. (2009) for the single zone model, it is assumed that the spray injected into the granulation zone gives rise to two different effects. The fraction of is converted into internal nuclei via overspray; the remaining fraction of leads to the layer-wise growth.

Assuming that the fraction of injected spray contributing to particle growth is equally distributed on the surface of all particles in the granulation zone, by following Mörl et al. (2007) and Vreman et al. (2009), the growth rate can be calculated as:

where represents the order moment of the number density .

It is assumed that the sizes of the nuclei formed from the overspray fraction of are normally distributed such that their normalized number-based particle size distribution is given by:

where stands for the mean diameter of the nuclei and for the standard deviation of the diameter. The particle flux of the nuclei from overspray is:

Following the arguments of Vreman et al. (2009), the fraction of contributing to overspray depends on the bed height, i.e. the path length that is available for small droplets to dry before they deposit upon the surface of the bed. Grünewald, Westhoff, and Kind (2010) proved this formulation by means of an experimental study. In addition, they showed that thermal and operational conditions also influence the nucleation. Nevertheless, a detailed consideration of thermal and operational conditions leading to nucleation is avoided. Instead, a simple linear relationship between bed height, nozzle height, and is used in the first approximation:

The parameter represents the minimum fraction of contributing to overspray if.

The product outlets, , in Fig. 2 enter a tube that is installed in the center of the bottom of the granulation chamber. Product classification is achieved with a counter-current stream of a classifying fluid, such as air. A detailed description and mathematical model of this internal product classification was given in Schmidt et al. (2015a) and Heinrich et al. (2002a) based on the work of 
Molerus and Hoffmann (1969). Here, we assume that the minimum diameter of the product particles withdrawn from the granulation chamber can be adjusted to a given value by manipulation of the classifying fluid.

For modeling the product removal, the withdrawal is described by:

approximating a Tromp curve (Rumpf, 1990; Stieß, 2009).

This is multiplied by a gain, which represents the ratio of the cross sections of the granulation chamber to the outlet tube. The function defines the probability of particles with size to be removed from the process with respect to the adjusted separation diameter and the sharpness of the separation. Since particles are withdrawn from both zones, the particle outlets of zones 1 and 2 are given by:

Neglecting attrition, breakage, and agglomeration, the dynamic behavior of the layering granulation process with internal product classification and nucleation is described by the population balances of the granulation and the drying zones according to Maronga and Wnukowski (1997), Wnukowski and Setterwall (1989), and Li, Freireich, Wassgren, and Litster (2012):

In this model, zone formation is described by the volumes of the granulation and the drying zones, and, and the characteristic time constants and. These quantities require careful consideration, since not all of them are independent, but they are related by the overall balances of the granulator. Characteristic values depend on the operating conditions, the nozzle and its position, according to the construction of the granulation chamber (Bück, Palis, \& Tsotsas, 2015). In contrast with processes with external classification and constant bed mass (Dreyschultze et al., 2015), the relative volume of the functional zones is expressed by the quantity, which is not constant because of the variable bed height/mass and the nucleation mechanism described above. Instead, we fix the absolute volume of the granulation zone and the characteristic time constant. Different values of these parameters are considered, to cover a wide range of possible process configurations. The volume of the drying zone and the characteristic time constant are then calculated from the overall balances.

The characteristic time constant follows from an overall mass balance of the granulation zone. For constant particle density, constant volume, and constant characteristic time the following relation is obtained:

where is the third order moment of the particles in the drying zone and is the third order moment of the product particles removed from the granulation zone via the classifying product removal. Bed height is obtained from: 
is the cross sectional area of the granulation chamber and is the total bed volume according to:

For some operating conditions, the number of particles in the granulator is too low to fill the given volume of the granulation zone with the given bed porosity. In these cases, the volume of the granulation zone is set to:

and the volume of the drying zone is equal to zero.

\section{Results and discussion}

In the following, the dynamics and stability of the FBLG process with internal product classification is analyzed numerically using the mathematical model developed in the previous section. For this purpose, numerical methods for dynamic simulation, parameter continuation, and stability analysis as introduced in Dreyschultze et al. (2015) are applied.

Model parameters are given in Table 1, comprising some common process parameters and three different sets of parameter pairs for the characterization of zone formation. Homogeneous behavior is observed if granulation takes place in the entire bed, or if mixing between the granulation and the drying zone, as characterized by in the present study, tends to infinity. A large value of also leads to a large value of according to the overall mass balance of the granulation zone, described in Eq. (9). Accordingly, parameter set in Table 1 represents a process with weak zone formation and parameter set is a process with strong zone formation. Set represents intermediate zone formation.

Table 1

Besides, the common parameters are consistent with Vreman et al. (2009) and Palis and Kienle (2013). is the primary bifurcation parameter in the present study.

\subsection{Influence of the injection rate}

The role of on process stability is illustrated in Fig. 3 for parameter set . Initial conditions correspond to the given reference value of in Table $1 \mathrm{of} 0.18 \mathrm{dm}^{3} / \mathrm{s}$; this corresponds to a stable steady state, no change of characteristic variables like, , mean diameter and the particle size distributions and was observed.

Fig. 3

At the injection rate was reduced to $0.17 \mathrm{dm}^{3} / \mathrm{s}$. A reduced amount of injected suspension yielded a decreasing growth rate of the particles. A reduction of the volume of the bed was thus observed and the height of the bed thus decreased. Although the volume of the bed changed, the absolute volume of the granulation zone was held constant. Because the drying zone changed in volume, the relative volume of the granulation zone increased. Since the bed height was larger than the height of the nozzle, the overspray fraction remained at, again leading to a stable steady state, which was attained after a transient period of a few hours in Fig. 3. The corresponding particle size distributions in the granulation and the drying zone are shown in Fig. 4 on the left. It is 
worth noting that the effect of on the particle size distribution of the granulation zone was barely noticeable because of the constant volume of the granulation zone. The effect on was larger.

Fig. 4

A further reduction of the injection rate to $0.16 \mathrm{dm}^{3} / \mathrm{s}$ at in Fig. 3 led to a significant change of the qualitative dynamic behavior. The steady state became unstable and all variables started oscillating with a period of about $7 \mathrm{~h}$. This is also seen in Fig. 4 on the right for the particle size distributions of the granulation and the drying zone. Vreman et al. (2009) and Palis and Kienle (2013) argued that these oscillations are caused by the negative feedback between the height of the bed and the overspray fraction. As Fig. 3 shows, the reduction decreased the total volume of the bed. While bed porosity was assumed to be constant, the height of the bed sank below the nozzle height ; consequently, the overspray fraction increased. This leads, as shown in Fig. 4, to an increased production of nuclei. While particles within the bed were growing, the height of the bed increased and exceeded the height of the nozzles. Consequently, the overspray fraction decreased to its minimum and the production of nuclei was reduced. Because of growth, the resulting peaks of the particle distributions and in Fig. 4 shifted toward higher particle sizes. When the peaks reached the separation diameter, a large number of product particles were withdrawn from the bed and the height of the bed dropped below the nozzle height. So, the production of nuclei rose and peaked in the particle distributions, and reappeared, initiating a new cycle of this periodic behavior.

\subsection{Influence of zone formation}

In this section, the influence of zone formation on stability is explored in more detail, with one parameter continuation of . A comparison is shown in Fig. 5 between parameter sets corresponding to a weak zone formation, and corresponding to a strong zone formation. Stable steady states of the FBLG process with internal product classification are represented by the solid lines at high injection rates. In this range, , so that attained the minimum value and nucleation does not depend on bed height. Steady states became unstable for corresponding to the dashed dotted lines in Fig. 5, because of the negative feedback between nucleation and bed height described previously. In this range, instability of the steady state leads to self-sustained oscillations. The amplitude of these oscillations is marked by the circles in Fig. 5. The amplitudes grew continuously from zero at the critical point, in the direction of decreasing injection rate. The influence of zone formation was moderate, as can be seen from comparison of the upper diagram (parameters set, weak zone formation) with the lower diagram (parameter set, strong zone formation). In the lower diagram the critical value of with shifted slightly to lower values compared with the upper diagram. The reason for this becomes obvious in Fig. 6, where the influence of zone formation on the overall steady state particle size distribution is shown for a stable steady state at $\mathrm{dm}^{3} / \mathrm{s}$. A comparison is given between a single zone model as proposed by Vreman et al. (2009) and a two-zone model with parameter sets and, respectively. It is shown that the total number of particles in the bed, corresponding to the area below the curves and therefore 
the bed height, increased with increasing zone formation and thereby shifted the critical value where to the left in Fig. 5. This can be explained in the following way. With increasing influence of zone formation, the size of the granulation zone decreases. So, for a given amount of injected liquid, the number of particles in the granulation zone will decrease but their size will increase. Hence, the total number of particles removed through the internal product classification will also be reduced, leading to a larger number of particles in the bed. The total number of particles in the bed corresponds to the zeroth order moments. In contrast, the third order moment, which is proportional to the total volume of particles removed from the bed, is fixed at steady state to the value of by the total mass balance of the granulation.

Figs. $5 \& 6$

\subsection{Influence of separation diameter}

Besides, the separation diameter (describing the minimum size of withdrawn particles ) was found to have a major influence on process stability. This is illustrated in Fig. 7 for parameter set by means of stability maps generated from two parameter continuations with variables and, using the approach described in Dreyschultze et al. (2015). Local stability was determined from the eigenvalues of the Jacobian of the linearization at the corresponding steady state. Parameter combinations with unstable steady states leading to self-sustained oscillations are indicated by the shaded region in Fig. 7; from above, the shaded region is bounded by the curve (red). In the upper right region we find, and hence . In this region, nucleation did not depend on bed height, which is a sufficient condition for stability. The upper stability boundary was also illustrated in Vreman et al. (2009) as well as in Palis and Kienle (2013). From below, the shaded region is bounded by a curve (blue) of supercritical Hopf bifurcation points (Thompson \& Stewart, 2002) with . Along this curve the steady state bed height increases continuously from the left to the right until equals at the point of intersection with the upper curve. The effect of the lower stability boundary is illustrated with a series of dynamic simulations in Fig. 8 similar to Fig. 3 for the upper boundary. In the left column, $\mathrm{mm}$. After two stepwise reductions of the shaded region is entered, as illustrated in Fig. 7, leading to instability in the form of self-sustained oscillations after time, when is set to $0.12 \mathrm{dm}^{3} / \mathrm{s}$. The bed height of the unstable steady state around which the system is oscillating (not shown in Fig. 8) is clearly below . However, during the oscillation the bed height also rose for a part of the period above, leading to a constant value of in this time interval, as shown in the lower diagram; in contrast, in the right column of Fig. 8 . Fig. 7 illustrates that a stepwise reduction of will lead to a stable steady state corresponding to a damped oscillation after (right column in Fig. 8).

Figs. $7 \& 8$

The shaded instability region in Fig. 7 is qualitatively in agreement with the experimental observations of Schmidt et al. (2015a); therein, a series of three experiments were reported with fixed and different values of the separation diameter, which was adjusted accordingly by manipulating the classifying air velocity of the product withdrawal. First, a stable steady state was found for a low separation diameter. After increasing the system became unstable and self- 
sustained oscillations were observed, corresponding to a crossing of the lower stability boundary. Afterward, the system returned to a stable steady state again after a further increase of corresponding to the crossing of the upper stability boundary.

\subsection{Influence of}

Finally, the influence of on process stability was studied. This is illustrated in Fig. 9 for parameter set by means of stability maps generated from two-parameter continuation with variables and. With reference to Fig. 7, the left diagram corresponds to the upper stability boundary with for a value of, the middle diagram represents the transition between the upper and the lower stability boundaries with for a value of, and the right diagram the lower stability boundary for a value of . Along the upper boundary, the critical value of increases for decreasing values of . This is because as decreases, the number of particles in the bed also decreases for along the upper stability boundary. Larger particles are thus required to fill the given volume with the given bed porosity. This can be achieved by increasing the injection rate . In contrast, the critical value of is almost constant along the lower stability boundary with and does not depend on. This is obvious since is greater than for all.

\section{Conclusion}

In this study a new population balance model for layering granulation processes with internal product classification and internal nucleation from overspray has been established. It assumes the formation of different functional zones, such that the granulation chamber is divided into a granulation and a drying zone. In contrast to previous models for batch (Hoffmann et al., 2011; Peglow et al., 2014; Silva et al., 2011) and continuous FBLG processes with external product classification (Dreyschultze et al., 2015), the relative size of the granulation zone is not constant anymore. This becomes clear from two limiting cases: (i) the volume of the spraying or granulation zone is restricted mainly to the region below the nozzle and hence will be similar for all bed heights above the nozzle height. In this case, only the volume of the drying zone will differ, (ii) for small bed heights the drying zone will vanish and almost all particles will be wetted by the injected liquid. Consequently, a constant volume granulation zone was assumed only for cases where the calculated bed volume was greater or equal to the given volume of the granulation zone. In contrast, if the calculated bed volume was lower than the given volume of the granulation zone, the actual volume of the granulation zone was adjusted accordingly and a vanishing volume for the drying zone was assumed.

The resulting model was used to investigate the influence of zone formation, and the most important operational parameters on the dynamic stability of the process. Zone formation has been found to have a moderate effect on process stability. In contrast to this, the stability depends crucially on the injection rate and the separation diameter, which can be adjusted by manipulating the classifying air flow rate of product withdrawal. Two different types of stability boundaries were found and previous theoretical (Vreman et al., 2009; Palis \& Kienle, 2013) and experimental 
findings (Schmidt et al., 2015a) could be explained consistently with these two different stability boundaries.

For this study, non-porous particles were assumed. Focus was on particle size as the characteristic product property. Other product properties like layer porosity may also be of interest from a practical point of view. It has recently been shown that particle porosity is strongly affected by zone formation (Rieck, Hoffmann, Bück, Peglow, \& Tsotsas, 2015). A simple, mechanistic model of the formation of new nuclei from overspray was applied, which neglected thermal conditions. The present model presents a promising basis for further model development to account for layer porosity or thermal conditions. Furthermore, it can be used for advanced applications in model-based control. Feedback control offers an interesting perspective for rejecting unforeseen disturbances and for the stabilization of fluidized bed layering granulation processes with internal (Palis \& Kienle, 2012a, 2013) and external product classification (Palis \& Kienle, 2012b, 2014).

\section{Acknowledgements}

We gratefully acknowledge the financial support of DFG (Deutsche Forschungsgemeinschaft) within the priority program SPP 1679.

\section{Notation}

cross sectional area $\left(\mathrm{m}^{2}\right)$

overspray fraction

minimum overspray fraction

$G \quad$ growth rate $(\mathrm{m} / \mathrm{s})$

bed height $(\mathrm{mm})$

nozzle height $(\mathrm{mm})$

gain of the withdrawal

particle size $(\mathrm{mm})$

average size of nuclei $(\mathrm{mm})$

average separation diameter $(\mathrm{mm})$

particle size distribution in zone $i(1 / \mathrm{mm})$

normalized particle size distribution in zone $i(1 / \mathrm{mm})$

time (s)

separation function

solid fraction of injected suspension $\left(\mathrm{dm}^{3} / \mathrm{s}\right)$

volume of zone $i\left(\mathrm{~m}^{3}\right)$

total bed volume $\left(\mathrm{m}^{3}\right)$

Greek letters

$\alpha \quad$ relative size of granulation zone

bed porosity

$i^{\text {th }}$ order moment of argument

parameter sets

standard deviation of nuclei size $(\mathrm{mm})$

standard deviation of separation diameter $(\mathrm{mm})$

\section{References}

residence time of particles (s)

Bück, A., Neugebauer, C., Meyer, K., Palis, S., Diez, E., \& Kienle, A., et al. (2016). Influence of operation parameters on process stability in continuous fluidised bed layering with external product classification. Powder Technology, accepted for publication. 
Bück, A., Palis, S., \& Tsotsas, E. (2015). Model-based control of particle properties in fluidised bed spray granulation. Powder Technology, 270, 575-583.

Dreyschultze, C., Neugebauer, C., Palis, S., Bück, A., Tsotsas, E., \& Heinrich, S., et al. (2015). Influence of zone formation on stability of continuous fluidized bed layering granulation with external product classification. Particuology, 23, 1-7.

Grünewald, G., Westhoff, B., \& Kind, M. (2010). Fluidized bed spray granulation: Nucleation studies with steady-state experiments. Drying Technology, 28(3), 349-360.

Heinrich, S., Peglow, M., Ihlow, M., Henneberg, M., \& Mörl, L. (2002a). Analysis of the start-up process in continuous fluidized bed spray granulation by population balance modelling. Chemical Engineering Science, 57(20), 4369-4390.

Heinrich, S., Peglow, M., \& Mörl, L. (2002b). Unsteady and steady-state particle size distributions in batch and continuous fluidized bed granulation systems. Chemical Engineering Journal, 86(1), 223-231.

Hoffmann, T., Peglow, M., \& Tsotsas, E. (2011). Prozessdynamik der Wirbelschichtgranulierung. Chemie Ingenieur Technik, 83(5), 658-664.

Li, J., Freireich, B., Wassgren, C., \& Litster, J. D. (2012). A general compartment-based population balance model for particle coating and layered granulation. AIChE Journal, 58(5), 13971408.

Maronga, S. J., \& Wnukowski, P. (1997). Establishing temperature and humidity profiles in fluidized bed particulate coating. Powder Technology, 94(2), 181-185.

Molerus, O., \& Hoffmann, H. (1969). Darstellung von Windsichtertrennkurven durch ein stochastisches Modell. Chemie Ingenieur Technik, 41(5-6), 340-344.

Mörl, L., Heinrich, S., \& Peglow, M. (2007). Fluidized bed spray granulation. In Handbook of powder technology (Vol. 11, pp. 21-188).

Palis, S., \& Kienle, A. (2012a, July). Discrepancy based control of continuous fluidized bed spray granulation with internal product classification. In Proceedings of 8th IFAC International Symposium on Advanced Control of Chemical Processes (pp. 756-761). Singapore.

Palis, S., \& Kienle, A. (2012b). Stabilization of continuous fluidized bed spray granulation with external product classification. Chemical Engineering Science, 70, 200-209.

Palis, S., \& Kienle, A. (2013). H-infinity loop shaping control for continuous fluidized bed spray granulation with internal product classification. Industrial \& Engineering Chemistry Research, 52(1), 408-420.

Palis, S., \& Kienle, A. (2014). Discrepancy based control of particulate processes. Journal of Process Control, 24(3), 33-46.

Peglow, M., Antonyuk, S., Jacob, M., Palzer, S., Heinrich, S., \& Tsotsas, E. (2014). Particle formulation in spray fluidized beds. In E. Tsotsas \& A. S. Mujumdar (Eds.), Modern Drying Technology (Vol. 3, pp. 295-378). 
Radichkov, R., Müller, T., Kienle, A., Heinrich, S., Peglow, M., \& Mörl, L. (2006). A numerical bifurcation analysis of continuous fluidized bed spray granulation with external product classification. Chemical Engineering and Processing, 45(10), 826-837.

Rieck, C., Hoffmann, T., Bück, A., Peglow, M., \& Tsotsas, E. (2015). Influence of drying conditions on layer porosity in fluidized bed spray granulation. Powder Technology, 272, $120-131$.

Rumpf, H. (1990). Particle technology. London: Chapman and Hall.

Schmidt, M., Bück, A., \& Tsotsas, E. (2015a). Experimental investigation of process stability of continuous spray fluidized bed layering with internal separation. Chemical Engineering Science, 126, 55-66.

Schmidt, M., Rieck, C., Bück, A., \& Tsotsas, E. (2015b). Experimental investigation of process stability of continuous spray uidized bed layering with external product separation. Chemical Engineering Science, 137, 466-475.

Schuette, R., Ruhs, A., Pelgrims, I., Klasen, C.-J., \& Kaiser, L. (1998). Fluidised bed spray granulation process producing two or more different size distributions. German Patent, DE 19639579.

Silva, D. O., Tamiozzo, L. M., Duarte, C. R., Murata, V. V., \& Barrozo, M. A. S. (2011). Modeling of seed coating in a spouted bed. Drying Technology, 29(3), 286-294.

Stieß, M. (2009). Mechanische Verfahrenstechnik - Partikeltechnologie 1. Berlin: Springer.

Thompson, J. M. T., \& Stewart, H. B. (2002). Nonlinear dynamics and chaos. New York: John Wiley \& Sons.

Vreman, A. W., Van Lare, C. E., \& Hounslow, M. J. (2009). A basic population balance model for fluid bed spray granulation. Chemical Engineering Science, 64(21), 4389-4398.

Wnukowski, P., \& Setterwall, F. (1989). The coating of particles in a fluidized bed (residence time distribution in a system of two coupled perfect mixers). Chemical Engineering Science, 44(3), 493-505. 


\section{Figures:}

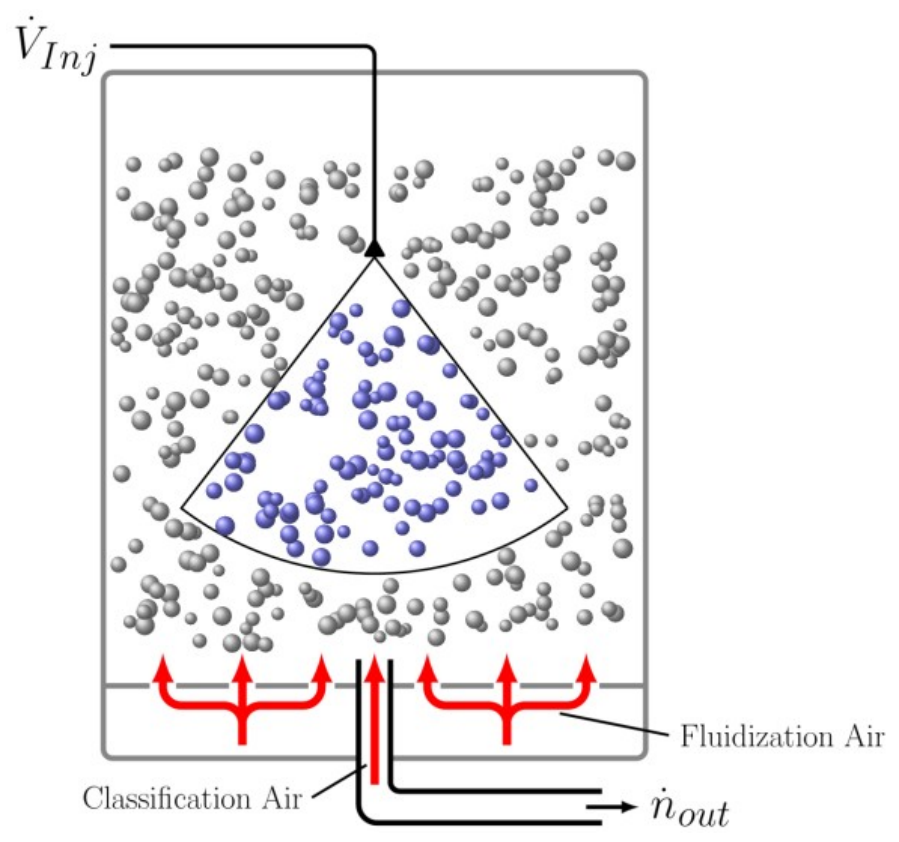

Fig. 1. Simplified scheme of fluidized bed layering granulation with internal product classification. The granulation zone is represented by the blue particles, the drying zone by the grey particles. 


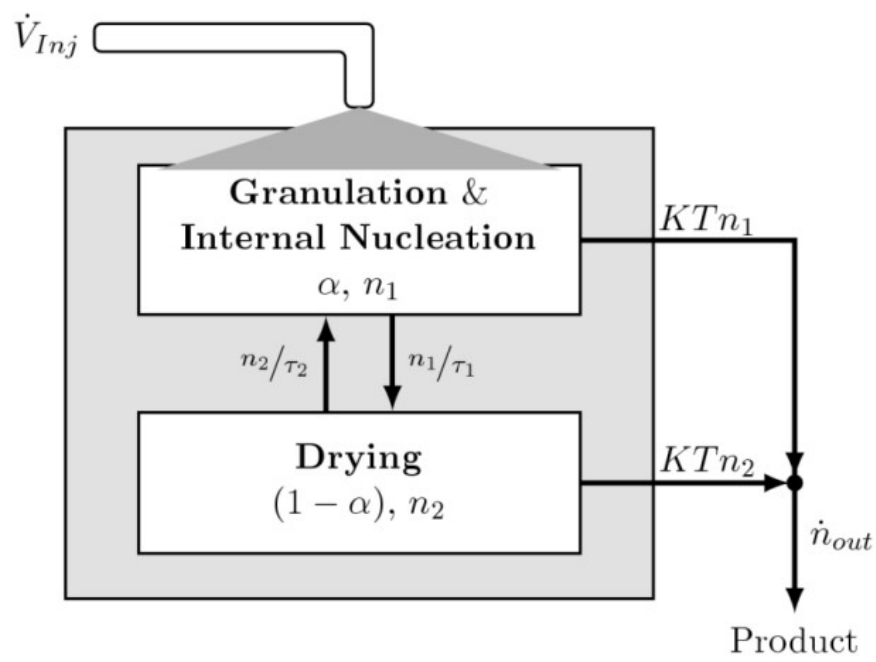

Fig. 2. Nomenclature and model structure of the fluidized bed layering granulation with internal product classification. 

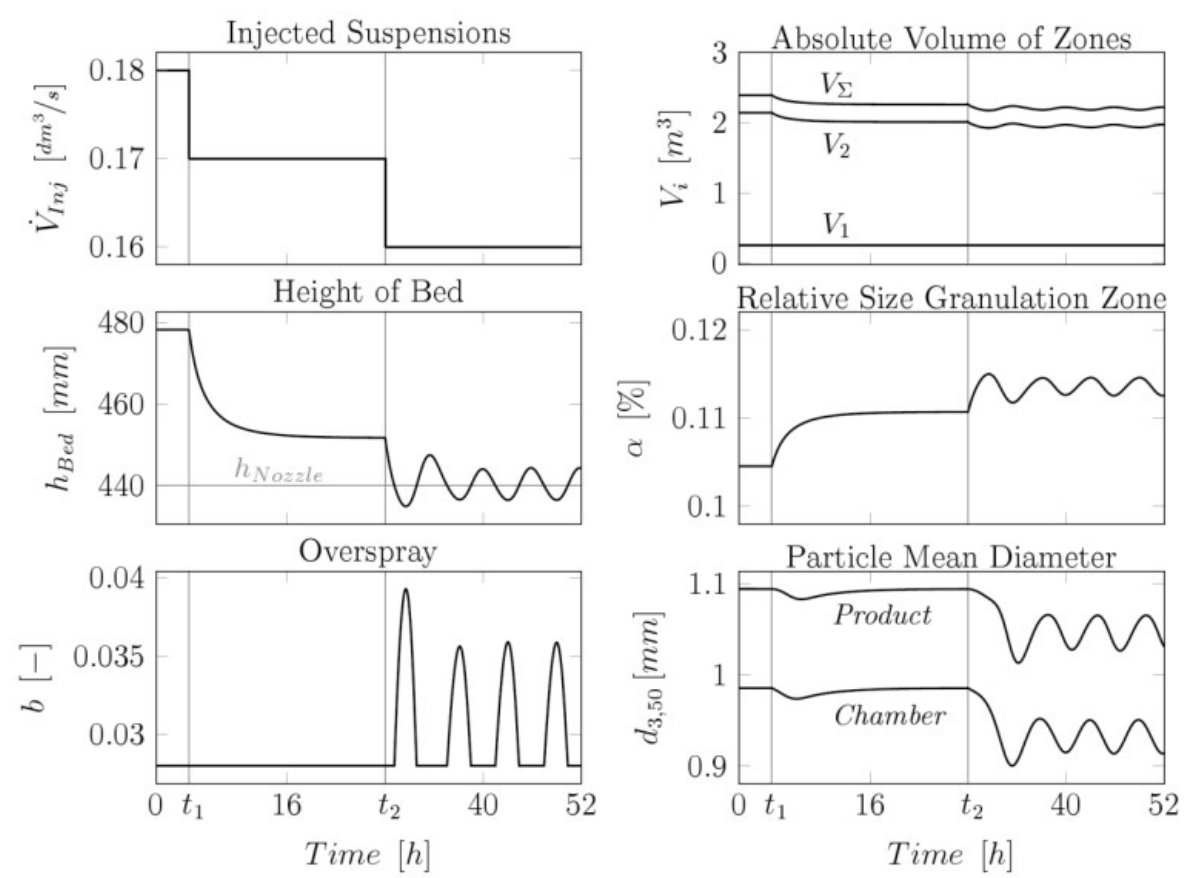

Fig. 3. Transient behavior of the layering granulation process with internal product classification

with parameter set $\Pi_{0}$. The injection rate is reduced to ${ }_{\mathrm{Inj}}^{\&}=0.17 \mathrm{dm} / \mathrm{s}$ and $\underset{\mathrm{Inj}}{\mathscr{\&}}=0.16 \mathrm{dm}^{3} / \mathrm{s}$ at $t_{1}=4 \mathrm{~h}$ and $t_{2}=28 \mathrm{~h}$, respectively. 

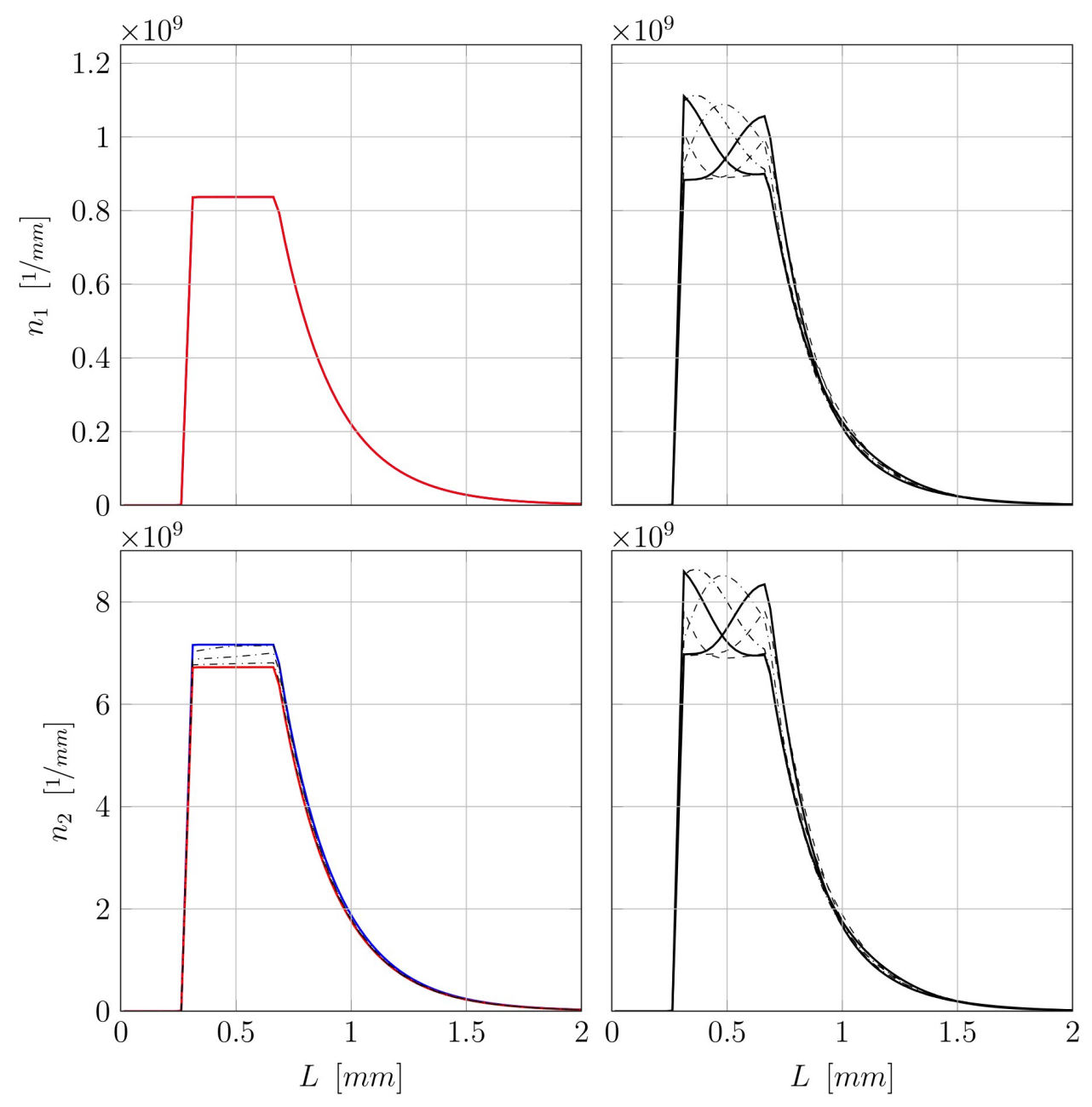

Fig. 4. Left column: transition of the particle size distributions $n_{1}$ and $n_{2}$ for a reduction of the injection rate from ${ }_{\text {Inj }}^{\&}=0.18 \mathrm{dm}^{3} / \mathrm{s}$ (blue) to $0.17 \mathrm{dm}^{3} / \mathrm{s}$ (red). Right column: limit cycle of $\boldsymbol{n}_{1}$ and $n_{2}$ for ${ }_{\mathrm{Inj}}^{\mathscr{\&}}=0.16 \mathrm{dm} / \mathrm{s}$; solid lines indicate particle size distribution $\boldsymbol{n}_{1}$ and $\boldsymbol{n}_{2}$ at minimum and maximum bed height $h_{\text {bed }}$ while dash dotted lines indicate the transition between these two particle size distributions. A detailed explanation is given in Section 3.1. 

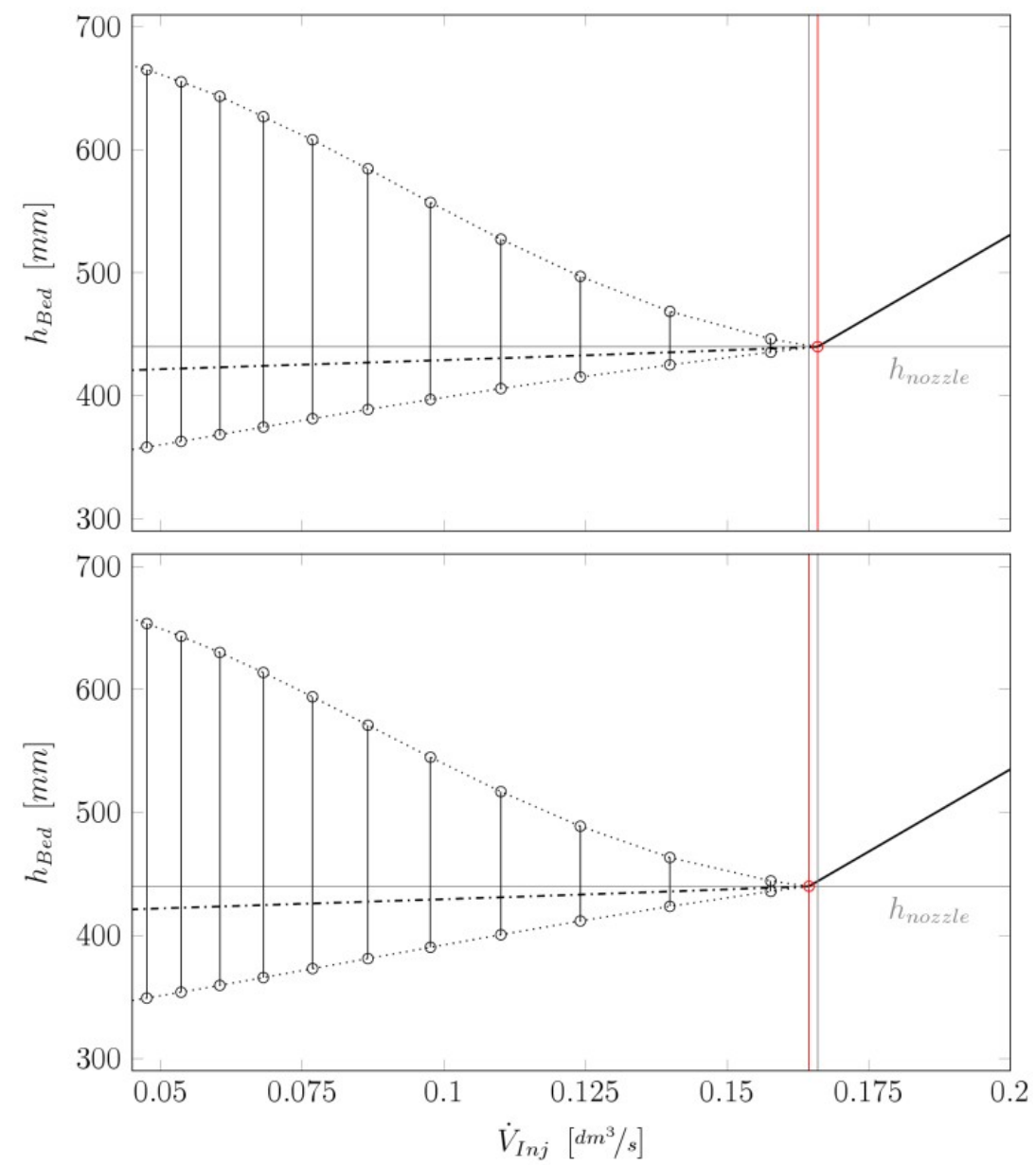

Fig. 5. Steady state and periodic solution branches for the bed height $h_{\text {bed }}$ in dependency of the injection rate $\stackrel{\mathrm{Inj}}{\mathrm{Inj}}_{\delta}$ for weak zone formation (top; parameter set $\Pi_{1}$ ) and strong zone formation (bottom; $\Pi_{2}$ ). The Hopf bifurcation points are indicated by a red line and a red circle. 


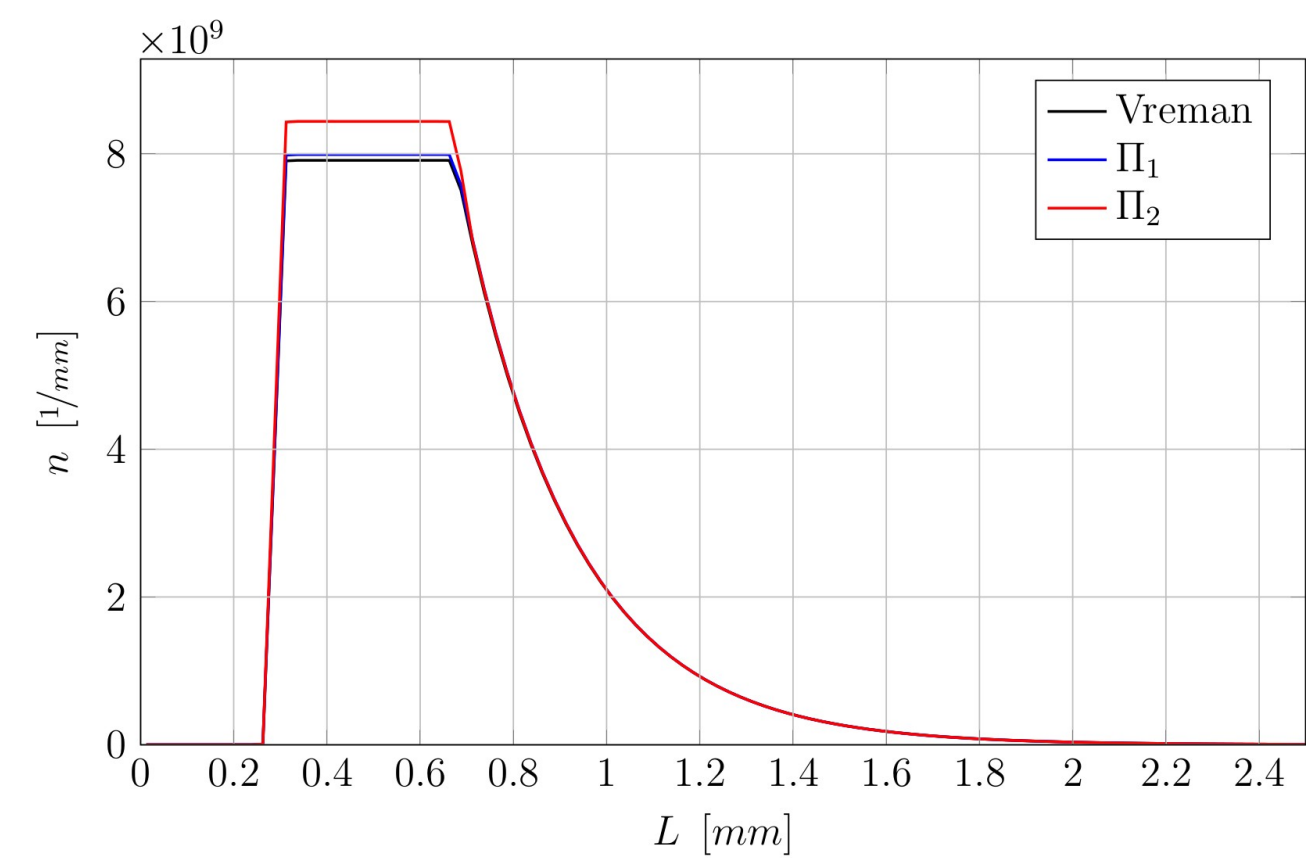

Fig. 6. Comparison of the steady state particle size distribution $n=\left(n_{1}+n_{2}\right)$ for parameter sets $\Pi_{1}, \Pi_{2}$ and the model given in Vreman et al. (2009) for $\mathscr{\mathrm { Inj }}_{\mathrm{L}}^{\alpha}=0.18 \mathrm{dm}^{3} / \mathrm{s}$. 


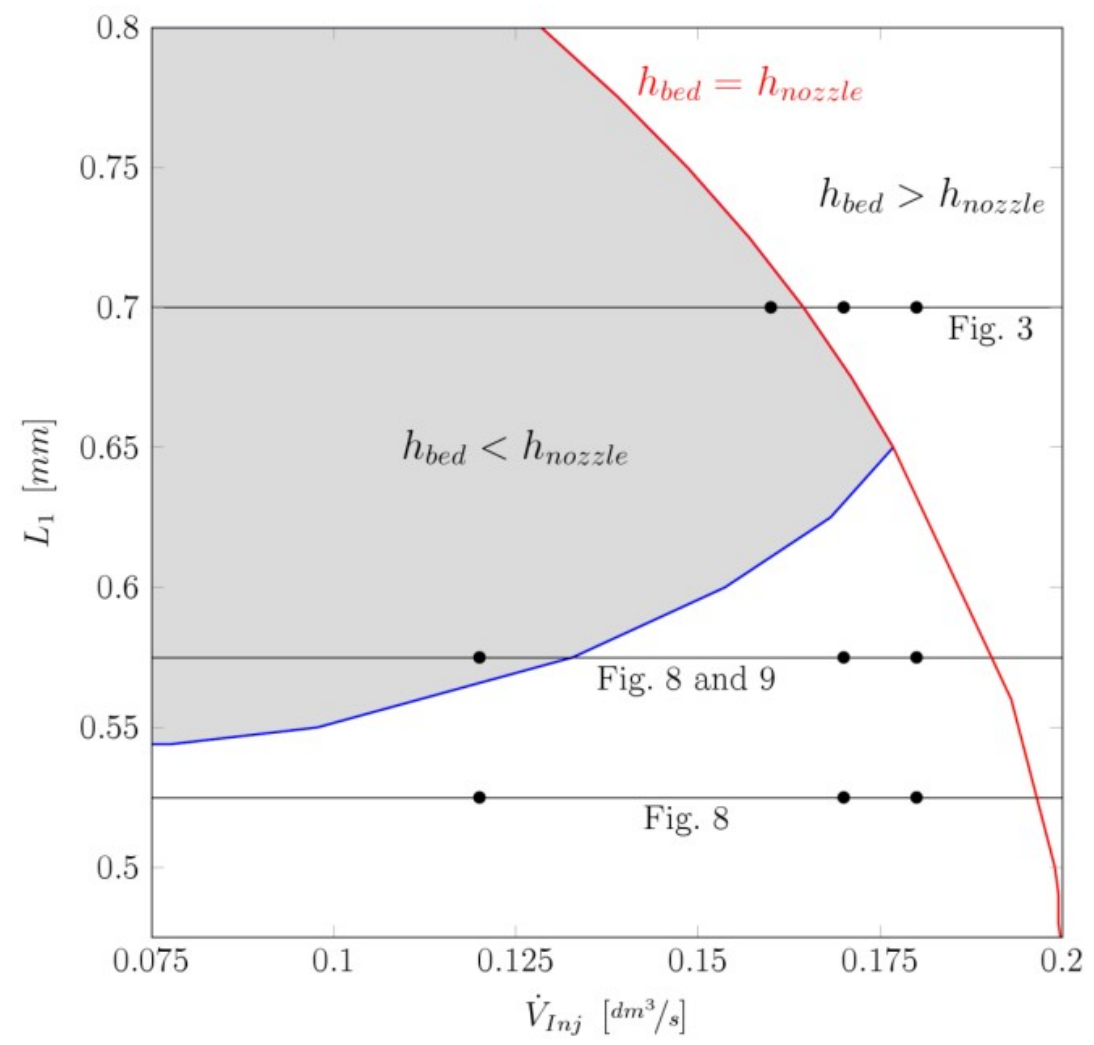

Fig. 7. Stability region in the $L_{1}-V_{\text {Inj }}^{\&}$ parameter plane for strong zone formation characterized by parameter set $\Pi_{2}$. The shaded region corresponds to unstable steady states leading to selfsustained oscillations. 

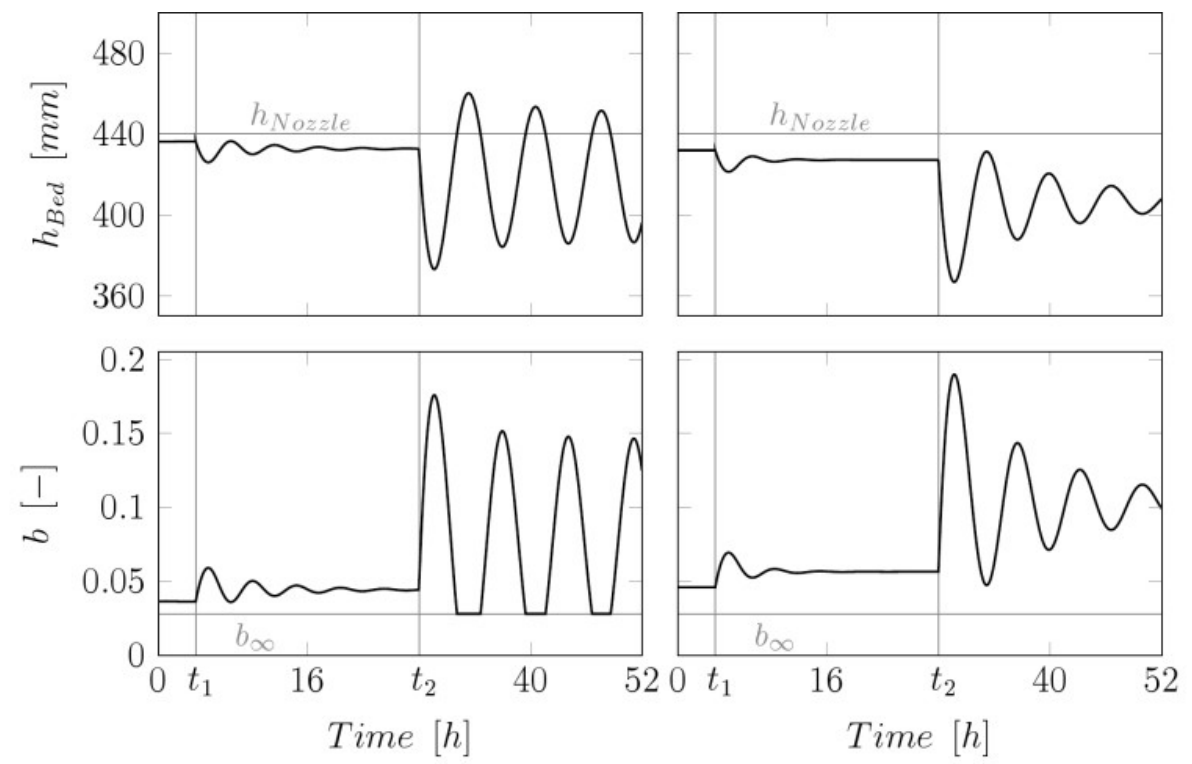

Fig. 8. Transient behavior of $h_{\text {bed }}$ and $b$ for $L_{1}=0.575 \mathrm{~mm}$ (left column) and $L_{1}=0.525 \mathrm{~mm}$ (right column) starting at $t=0 \mathrm{~h}$ with $t_{\mathrm{Inj}}^{\&}=0.18 \mathrm{dm}^{3} / \mathrm{s}$. At $t_{1}=4 \mathrm{~h}$ and $t_{2}=28 \mathrm{~h}$ the injection rate was reduced to ${ }_{\mathrm{Inj}}^{\&}=0.17 \mathrm{dm}^{3} / \mathrm{s}$ and $L_{\mathrm{Inj}}^{\alpha}=0.12 \mathrm{dm}^{3} / \mathrm{s}$, respectively. Parameter set $\Pi_{2}$, representing a strong zone formation, is used, compared to Fig. 7. 

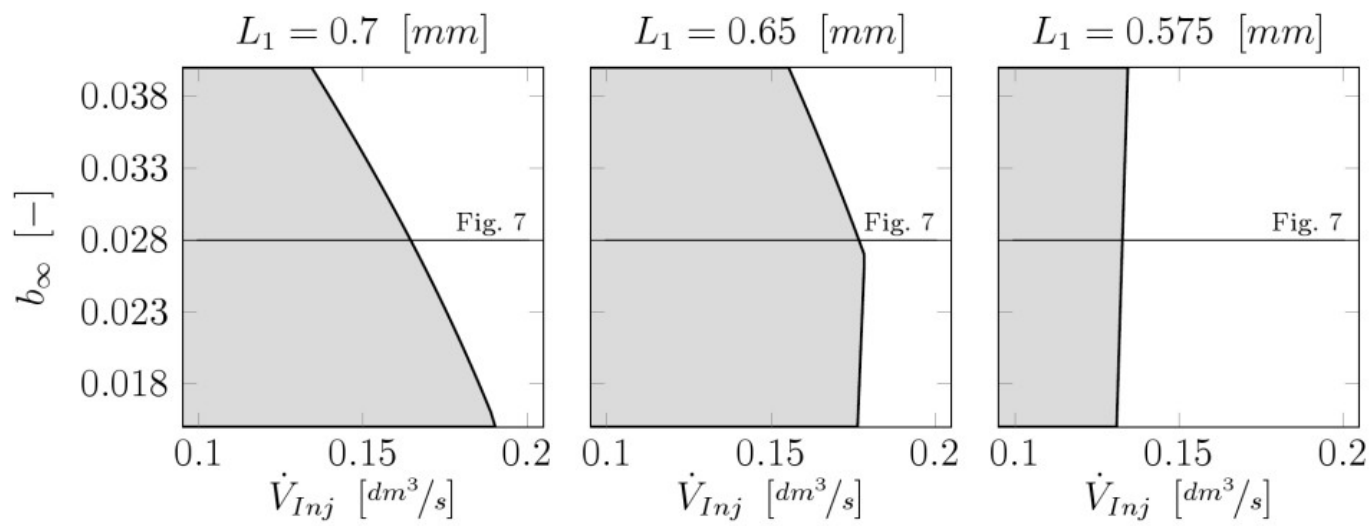

Fig. 9. Influence of minimum overspray fraction $b_{\square}$ on dynamic stability in the $b_{\square}-L_{\mathrm{Inj}}^{\mathcal{\alpha}}$ parameter plane for strong zone formation (parameter set $\Pi_{2}$ ). 


\section{Table}

Table 1. Common process parameters for the FBLG and parameter sets to investigate the influence of zone formation on dynamic stability

\begin{tabular}{|c|c|c|}
\hline \multicolumn{3}{|c|}{ Process parameter ( $\star$ as reported in (Vreman et al., 2009)) } \\
\hline \multicolumn{3}{|c|}{ Granulation chamber } \\
\hline$A \overline{\left(\mathrm{m}^{2}\right)}$ & 5.00 & * \\
\hline$V_{1}\left(\mathrm{~m}^{3}\right)$ & 0.25 & \\
\hline$\tau_{2} \quad(\mathrm{~s})$ & 60.00 & \\
\hline $\mathscr{I I n j}_{\left(\mathrm{dm}^{3} / \mathrm{s}\right)}^{\mathcal{E}}$ & 0.18 & \\
\hline$h_{\text {nozzle }}(\mathrm{mm})$ & 440.00 & * \\
\hline$\varepsilon$ & 0.50 & * \\
\hline \multicolumn{3}{|l|}{ Internal nuclei } \\
\hline$L_{0} \quad(\mathrm{~mm})$ & 0.30 & 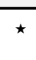 \\
\hline$\sigma_{0} \quad(\mathrm{~mm})$ & 0.01 & \\
\hline$b_{\infty}$ & 0.028 & * \\
\hline \multicolumn{3}{|l|}{ Withdrawal } \\
\hline$L_{1} \quad(\mathrm{~mm})$ & 0.70 & * \\
\hline$\sigma_{1}(\mathrm{~mm})$ & 0.01 & \\
\hline$K$ & $1.92 \times 10^{-4}$ & * \\
\hline \multicolumn{3}{|c|}{ Parameter sets for zone formation } \\
\hline \multicolumn{3}{|c|}{ Default parameter set $\Pi_{0}$} \\
\hline$V_{1}\left(\mathrm{~m}^{3}\right)$ & 0.25 & \\
\hline$\tau_{2} \quad(\mathrm{~s})$ & 60.00 & \\
\hline \multicolumn{3}{|c|}{ Weak zone formation $\Pi_{1}$} \\
\hline$V_{1}\left(\mathrm{~m}^{3}\right)$ & 2.10 & \\
\hline
\end{tabular}




\begin{tabular}{|c|r|l|}
\hline$\tau_{2}(\mathrm{~s})$ & 10.00 & \\
\hline \multicolumn{2}{|l|}{ Strong zone formation $\Pi_{2}$} & \\
\hline$V_{1}\left(\mathrm{~m}^{3}\right)$ & 0.001 & \\
\hline$\tau_{2}(\mathrm{~s})$ & 300.00 & \\
\hline
\end{tabular}




\section{Highlights:}

- Dynamic population balance model of continuous fluidized bed layering granulation with distinct drying and granulation zones was developed.

- Stability was studied for a wide range of possible process configurations and operating conditions.

- Results were validated by dynamic simulation.

- Previous experimental findings were reproduced and justified.

\section{Graphical Abstract:}
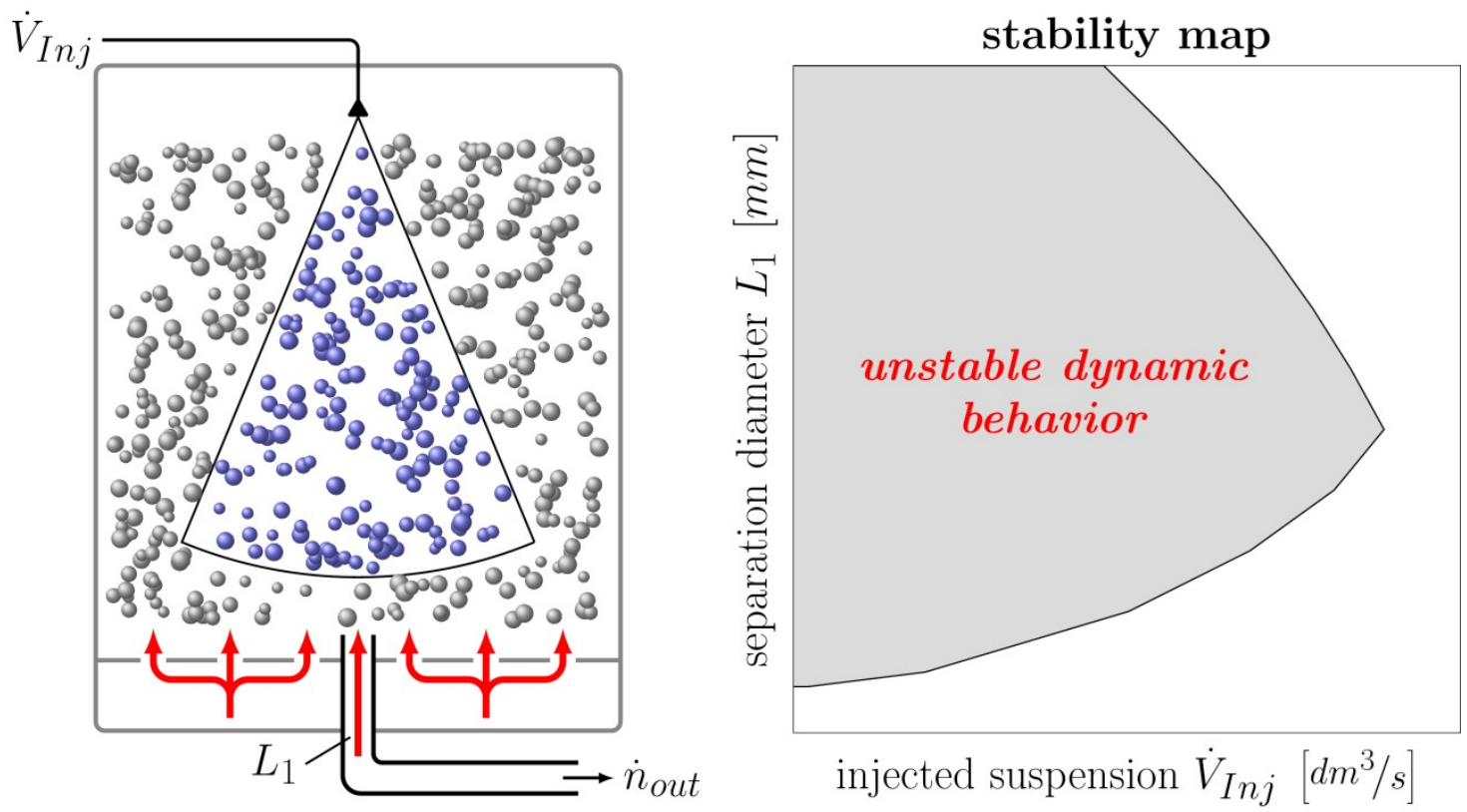